\title{
Escherichia coli Isolated from Urinary Tract Infections of Lebanese Patients between 2000 and 2009: Epidemiology and Profiles of Resistance
}

\author{
Ziad Daoud $^{1}$ and Claude Afif ${ }^{2}$ \\ ${ }^{1}$ Faculty of Medicine and Medical Sciences, University of Balamand, P.O. Box 100, Tripoli, Lebanon \\ ${ }^{2}$ Faculty of Medicine and Medical Sciences, University of Balamand, P.O. Box 166378, Beirut, Lebanon \\ Correspondence should be addressed to Ziad Daoud, ziad.daoud@balamand.edu.lb
}

Received 1 July 2011; Revised 10 August 2011; Accepted 13 August 2011

Academic Editor: Spyros Pournaras

Copyright ( $) 2011$ Z. Daoud and C. Afif. This is an open access article distributed under the Creative Commons Attribution License, which permits unrestricted use, distribution, and reproduction in any medium, provided the original work is properly cited.

\begin{abstract}
The purpose of this study was to investigate the bacterial etiology of urinary tract infections in one of the busiest hospitals of Lebanon and to examine the epidemiologic and microbiologic properties of Escherichia coli isolated from urinary tract infections of Lebanese patients over a 10-year period. Methods. This retrospective study analyzed the data generated between 2000 and 2009 (10,013 Gram-positive and Gram-negative bacteria). Bacterial identification was based on standard culture and biochemical characteristics of isolates. Antimicrobial susceptibility was tested by the disk diffusion method, and ESBL production was detected by synergy with third-generation cephalosporins and amoxiclav. Results. E. coli was the most frequent isolate throughout the ten years (60.64\% of the total isolates). It was followed by Klebsiella pneumoniae and Proteus sp., Pseudomonas aeruginosa, Enterococcus sp., and Streptococcus agalactiae. E. coli occurred more frequently in women (69.8\%) than in men (61.4\%). The lowest percentage of susceptibility of $E$. coli was manifested against piperacillin and ampicillin. An increase in the production of ESBL was observed (2.3\% in 2000 to $16.8 \%$ in 2009). Conclusions. The etiology of urinary tract infections and their susceptibility profiles are important to be evaluated in countries like Lebanon where a severe misuse of antibiotics at all levels is observed.
\end{abstract}

\section{Introduction}

Urinary tract infections (UTIs) are one of the most common infectious diseases [1-3]. They may be symptomatic or asymptomatic, and either type of infection can result in serious sequelae if not appropriately treated [4]. Although different causative agents can be responsible for UTIs, bacteria are the major cause being responsible for more than $95 \%$ of UTI cases [5]. In this context, E. coli is the most prevalent organism and is solely responsible for the majority of these infections. An accurate and prompt diagnosis is important in shortening the disease course and for preventing the ascent of the infection to the upper urinary tract [6]. Treatment of UTI is often started empirically. UTIs are often treated with different broad-spectrum antibiotics when one with a narrow spectrum of activity may be appropriate because of concerns about infection with resistant organisms, and antimicrobial susceptibility testing of the urinary pathogens constitutes the basis for antibiotic therapy. However, in view of the increasing bacterial resistance, regular monitoring of resistance patterns is necessary to improve guidelines for empirical antibiotic therapy [6-8]. The present study aims at analyzing the infectious epidemiology of UTIs in a general university hospital located in Beirut over a period of ten years. In addition, it examines the susceptibility profiles of E. coli between 2000 and 2009.

\section{Material and Methods}

2.1. Study Design. This is a retrospective study conducted at the Microbiology section of the Medical Laboratories of the Saint George Hospital-University Medical Center in Beirut and covering ten years (2000 to 2009). The population included all in- and outpatients with documented UTI. This included 10,013 different Gram-positive and Gram-negative bacteria in addition to Candida albicans and Candida sp. 
There were $6,708(66.99 \%)$ samples from female patients and 3,305 (33.01\%) from male patients. Adult patients were sampled by clean catch midstream urine, and children aged less than 3 years were sampled using sterile urine bags. Only a single positive culture per patient was included in the analysis within the period of three months.

2.2. Isolation and Identification of Organisms. Samples for urine culture were tested within half an hour of sampling. All samples were inoculated on blood agar as well as Mac Conckey agar and incubated at $37^{\circ} \mathrm{C}$ for 24 hours, and for 48 hours in negative cases. A specimen was considered positive for UTI in the light of the number of yielded colonies $\left(\geq 10^{5} \mathrm{cfu} / \mathrm{mL}\right)$ and the cytology of the urine through microscopic detection of bacteriuria and PMNs $(\geq 8$ leukocytes $/ \mathrm{mm}^{3}$ ). However, lower colony counts associated with significant pyuria or low PMN count associated with significant colony counts was considered and analyzed in the light of the clinical picture and the patient's immunological status. Bacterial identification was based on standard culture and biochemical characteristics of isolates.

Gram-negative bacteria were identified by standard biochemical tests $[5,6]$. Gram-positive microorganisms were identified with the corresponding recommended laboratory tests.

2.3. Susceptibility Testing. Antimicrobial susceptibility of E. coli was tested by the disk diffusion method according to the CLSI recommendations, using the Mueller-Hinton agar [6]. Antimicrobial agents tested were ampicillin, amoxicillin-clavulanic acid, aztreonam, cephalothin, cefoxitin, cefuroxime, cefotaxime, ceftriaxone, ceftazidime, cefipime, piperacillin, piperacillin-tazobactam, imipenem, gentamycin, tobramycin, norfloxacin, ciprofloxacin, trimethoprim-sulfamethoxazole, and tetracycline. The CLSI-ESBL phenotypic confirmatory test with ceftazidime, cefotaxime, ceftriaxone, and cefixime was performed for all the isolates by disk diffusion method on the Mueller-Hinton agar plates with and without $10 \mu \mathrm{g}$ of amoxiclav. Susceptibility test results were interpreted according to the criteria established by the Clinical \& Laboratory Standard Institute (CLSI). A minimum of $5 \mathrm{~mm}$ increase in the zone of diameter of third-generation cephalosporins, tested in combination with amoxiclav versus its zone when tested alone, was considered indicative of ESBL production. E. coli ATCC 25922 was used as ESBL-negative and K. pneumoniae 700603 was used as ESBL-positive reference strain. Statistical analysis: variables were expressed as percentages.

\section{Results}

Over a 10-year period, a total of 10,013 positive urine isolates including 6,071 E. coli were analyzed.

Tables 1 and 2 show the microorganisms most frequently isolated from these positive urine cultures. As expected, E. coli was the most frequent isolate throughout the ten years (average of $60.64 \%$ of the total isolates). It was followed by Klebsiella pneumoniae where rate of isolation ranged between
6.1 and $9.9 \%$. The next most frequently isolated bacteria were Proteus sp., Pseudomonas aeruginosa, Enterococcus sp., and Streptococcus agalactiae. Candida albicans and Candida sp. were commonly isolated; however, their clinical significance was not always evident. If Candida and minor bacterial isolates are not included, Gram-negative bacteria accounted for $92 \%$ of the UTI, while Gram-positive infections were responsible only for $8 \%$.

Analysis of the results according to patient gender (not shown) indicated that although $E$. coli is the predominant isolated pathogen from both sexes, it occurred more frequently in women $(69.8 \%$ in women compared to $61.4 \%$ in men.) The trend of infectious etiology in the population did not really differ over the 10 -year period.

The percentages of susceptibility (and subsequently of nonsusceptibility including both resistant and intermediately resistant strains) of $E$. coli isolates to the panel of antibiotics which are commonly used to treat Escherichia infections are shown in Table 3. The lowest percentage of susceptibility was manifested against piperacillin (between 9 and 24\%) followed by ampicillin (between 26 and 38\%), whereas an absolute susceptibility was observed with imipenem (100\%). In general, the urinary isolates showed a slightly better susceptibility profile in comparison to all the hospital isolates of E. coli. Over the successive years, the susceptibility to cephalosporins, including generations 3 and 4, tends to decrease; this is coupled by an increase in the production of ESBL as shown in Table 4 where ESBL production goes from $2.3 \%$ in 2000 to $16.8 \%$ in 2009 for the urinary isolates.

Table 5 shows the susceptibility profiles of ESBL producing E. coli to families of antibiotics other than beta-lactams. Tigecycline, amikacin, and piperacillin-tazobactam seem to have the highest antibacterial activity on these organisms.

Table 6 shows the percentages of simultaneous resistance in the urinary isolates of $E$. coli to various families of antimicrobial agents.

\section{Discussion}

This study shows the distribution of microbial species and antibiotic susceptibility patterns of $E$. coli isolated from Lebanese patients with UTIs. Saint George Hospital is a 300-bed hospital located in Beirut; however, it is one of the busiest hospitals in the country and receives patients from different areas of Lebanon. In this retrospective study, no clinical data are provided, and this constitutes by itself a limitation. Subsequently, important information related to symptomatic versus asymptomatic, complicated versus uncomplicated UTI, and health-care-associated versus catheter-related UTIs could not be addressed and discussed. The majority of pathogens were isolated from women $(69.8 \%)$. It has been extensively reported that adult women have a higher prevalence of UTI than men, principally owing to anatomic and physical factors [9].

Antibiotic resistance is a major clinical problem in treating infections caused by these microorganisms. The resistance to the antimicrobials has increased over the years. Resistance rates vary from country to country [10]. In Lebanon, there is an evidence for increase in ESBL producing 
TABle 1: The organisms most commonly isolated from Lebanese patients with UTI between 2000 and 2004 at Saint George Hospital University Medical Center, Beirut.

\begin{tabular}{|c|c|c|c|c|c|c|c|c|c|c|}
\hline & \multicolumn{10}{|c|}{ The microorganisms most commonly isolated from Lebanese patients with UTI between 2000 and 2004} \\
\hline & \multicolumn{2}{|c|}{2000} & \multicolumn{2}{|c|}{2001} & \multicolumn{2}{|c|}{2002} & \multicolumn{2}{|c|}{2003} & \multicolumn{2}{|c|}{2004} \\
\hline & $\mathrm{Nb}$ & $\%$ & $\mathrm{Nb}$ & $\%$ & $\mathrm{Nb}$ & $\%$ & $\mathrm{Nb}$ & $\%$ & $\mathrm{Nb}$ & $\%$ \\
\hline Acinetobacter sp. & 3 & 0.5 & 4 & 0.5 & 2 & 0.2 & 0 & 0.0 & 14 & 1.3 \\
\hline Candida albicans & 15 & 2.4 & 12 & 1.6 & 23 & 2.4 & 26 & 2.4 & 31 & 2.9 \\
\hline Candida sp. & 16 & 2.5 & 22 & 2.9 & 28 & 2.9 & 47 & 4.3 & 34 & 3.1 \\
\hline Citrobacter sp. & 6 & 0.9 & 11 & 1.5 & 19 & 2.0 & 17 & 1.5 & 15 & 1.4 \\
\hline Enterobacter sp. & 4 & 0.6 & 12 & 1.6 & 14 & 1.5 & 6 & 0.5 & 16 & 1.5 \\
\hline Enterococcus faecalis & 17 & 2.7 & 18 & 2.4 & 28 & 2.9 & 48 & 4.4 & 20 & 1.9 \\
\hline Enterococcus faecium & 6 & 0.9 & 18 & 2.4 & 20 & 2.1 & 30 & 2.7 & 35 & 3.2 \\
\hline Escherichia coli & 395 & 62.5 & 440 & 58.3 & 575 & 59.8 & 637 & 57.9 & 661 & 61.1 \\
\hline Klebsiella sp. & 48 & 7.6 & 75 & 9.9 & 59 & 6.1 & 85 & 7.7 & 79 & 7.3 \\
\hline Morganella morganii & 5 & 0.8 & 4 & 0.5 & 11 & 1.1 & 14 & 1.3 & 14 & 1.3 \\
\hline Proteus sp. & 45 & 7.1 & 50 & 6.6 & 72 & 7.5 & 74 & 6.7 & 65 & 6.0 \\
\hline Pseudomonas aeruginosa & 30 & 4.7 & 38 & 5.0 & 45 & 4.7 & 35 & 3.2 & 51 & 4.7 \\
\hline Pseudomonas sp. & 0 & 0.0 & 2 & 0.3 & 1 & 0.1 & 1 & 0.1 & 0 & 0.0 \\
\hline Staphylococcus aureus & 3 & 0.5 & 6 & 0.8 & 3 & 0.3 & 7 & 0.6 & 9 & 0.8 \\
\hline $\begin{array}{l}\text { Staphylococcus } \\
\text { saprophyticus }\end{array}$ & 6 & 0.9 & 7 & 0.9 & 5 & 0.5 & 2 & 0.2 & 3 & 0.3 \\
\hline Streptococcus agalactiae & 20 & 3.2 & 15 & 2.0 & 14 & 1.5 & 25 & 2.3 & 18 & 1.7 \\
\hline Streptococcus, Group D & 1 & 0.2 & 2 & 0.3 & 4 & 0.4 & 1 & 0.1 & 6 & 0.6 \\
\hline Other minor organisms & 12 & 1.9 & 19 & 2.5 & 38 & 4.0 & 45 & 4.1 & 10 & 0.9 \\
\hline Total isolates & \multicolumn{2}{|c|}{632} & \multicolumn{2}{|c|}{755} & \multicolumn{2}{|c|}{961} & \multicolumn{2}{|c|}{1100} & \multicolumn{2}{|c|}{1081} \\
\hline
\end{tabular}

TABle 2: The organisms most commonly isolated from Lebanese patients with UTI between 2000 and 2004 at Saint George Hospital University Medical Center, Beirut.

\begin{tabular}{|c|c|c|c|c|c|c|c|c|c|c|}
\hline & \multicolumn{10}{|c|}{ The microorganisms most commonly isolated from Lebanese patients with UTI between 2005 and 2009} \\
\hline & \multicolumn{2}{|c|}{2005} & \multicolumn{2}{|c|}{2006} & \multicolumn{2}{|c|}{2007} & \multicolumn{2}{|c|}{2008} & \multicolumn{2}{|c|}{2009} \\
\hline & $\mathrm{Nb}$ & $\%$ & $\mathrm{Nb}$ & $\%$ & $\mathrm{Nb}$ & $\%$ & $\mathrm{Nb}$ & $\%$ & $\mathrm{Nb}$ & $\%$ \\
\hline Acinetobacter sp. & 19 & 1.9 & 14 & 1.2 & 18 & 1.6 & 16 & 1.3 & 5 & 0.5 \\
\hline Candida albicans & 14 & 1.4 & 37 & 3.3 & 27 & 2.4 & 29 & 2.4 & 25 & 2.5 \\
\hline Candida sp. & 29 & 2.9 & 57 & 5.1 & 34 & 3.0 & 31 & 2.6 & 35 & 3.5 \\
\hline Citrobacter sp. & 15 & 1.5 & 18 & 1.6 & 19 & 1.7 & 16 & 1.3 & 9 & 0.9 \\
\hline Enterobacter sp. & 16 & 1.6 & 22 & 2.0 & 14 & 1.2 & 19 & 1.6 & 6 & 0.6 \\
\hline Enterococcus faecalis & 14 & 1.4 & 15 & 1.3 & 20 & 1.8 & 14 & 1.2 & 15 & 1.5 \\
\hline Enterococcus faecium & 32 & 3.2 & 25 & 2.2 & 34 & 3.0 & 34 & 2.8 & 6 & 0.6 \\
\hline Escherichia coli & 609 & 60.2 & 711 & 63.4 & 688 & 60.7 & 727 & 60.0 & 628 & 62.5 \\
\hline Klebsiella sp. & 92 & 9.1 & 84 & 7.5 & 100 & 8.8 & 118 & 9.7 & 90 & 9.0 \\
\hline Morganella morganii & 13 & 1.3 & 6 & 0.5 & 10 & 0.9 & 24 & 2.0 & 7 & 0.7 \\
\hline Proteus sp. & 57 & 5.6 & 53 & 4.7 & 70 & 6.2 & 73 & 6.0 & 63 & 6.3 \\
\hline Pseudomonas aeruginosa & 39 & 3.9 & 27 & 2.4 & 40 & 3.5 & 44 & 3.6 & 41 & 4.1 \\
\hline Pseudomonas sp. & 0 & 0.0 & 6 & 0.5 & 0 & 0.0 & 6 & 0.5 & 3 & 0.3 \\
\hline Staphylococcus aureus & 7 & 0.7 & 4 & 0.4 & 6 & 0.5 & 4 & 0.3 & 10 & 1.0 \\
\hline $\begin{array}{l}\text { Staphylococcus } \\
\text { saprophyticus }\end{array}$ & 0 & 0.0 & 5 & 0.4 & 1 & 0.1 & 2 & 0.2 & 3 & 0.3 \\
\hline Streptococcus agalactiae & 27 & 2.7 & 14 & 1.2 & 16 & 1.4 & 16 & 1.3 & 8 & 0.8 \\
\hline Streptococcus, Group D & 1 & 0.1 & 1 & 0.1 & 1 & 0.1 & 0 & 0.0 & 1 & 0.1 \\
\hline Other minor organisms & 28 & 2.8 & 23 & 2.0 & 36 & 3.2 & 38 & 3.1 & 50 & 5.0 \\
\hline Total isolates & \multicolumn{2}{|c|}{1012} & \multicolumn{2}{|c|}{1122} & \multicolumn{2}{|c|}{1134} & \multicolumn{2}{|c|}{1211} & \multicolumn{2}{|c|}{1005} \\
\hline
\end{tabular}




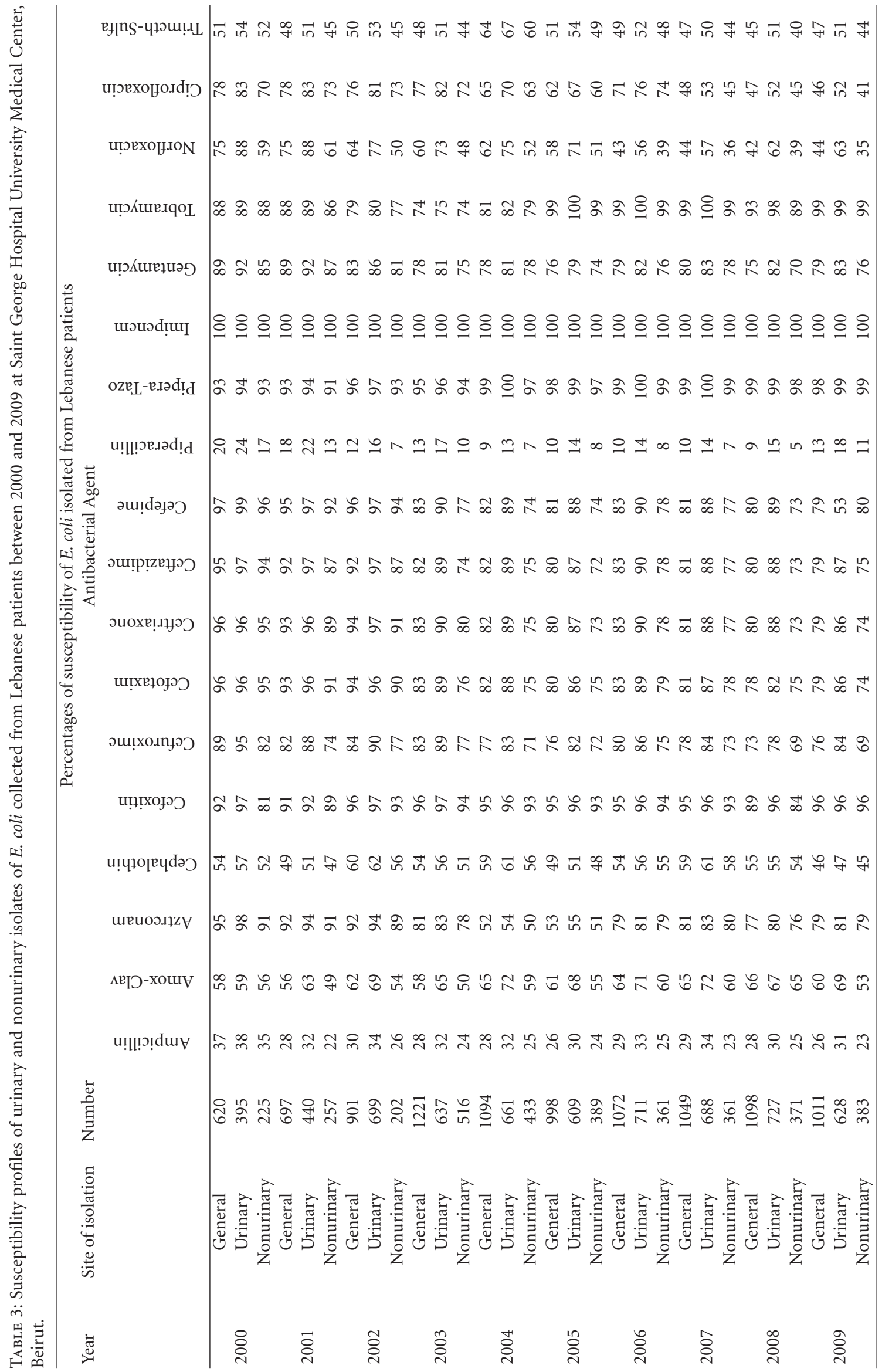


TABLE 4: ESBL production in E. coli collected from Lebanese patients between 2000 and 2009 at the Saint George Hospital University Medical Center, Beirut. U: urinary isolates, NU: nonurinary isolates.

\begin{tabular}{|c|c|c|c|c|c|c|c|c|c|c|c|c|c|c|c|}
\hline \multirow[b]{3}{*}{ Isolates } & \multicolumn{15}{|c|}{ ESBL production in E. coli isolated between 2000 and 2009} \\
\hline & \multicolumn{3}{|c|}{2000} & \multicolumn{3}{|c|}{2001} & \multicolumn{3}{|c|}{2002} & \multicolumn{3}{|c|}{2003} & \multicolumn{3}{|c|}{2004} \\
\hline & All & $\mathrm{U}$ & NU & All & $\mathrm{U}$ & NU & All & $\mathrm{U}$ & NU & All & $\mathrm{U}$ & NU & All & $\mathrm{U}$ & NU \\
\hline Number of isolates & 620 & 395 & 225 & 697 & 440 & 257 & 901 & 699 & 202 & 1221 & 637 & 584 & 1094 & 661 & 433 \\
\hline \multirow[t]{2}{*}{$\%$ of ESBL } & 2.3 & 2.1 & 2.3 & 4 & 3.9 & 4.3 & 9.8 & 9.9 & 9.5 & 13.6 & 14.5 & 12.1 & 12.9 & 11.0 & 14.1 \\
\hline & & 2005 & & & 2006 & & & 2007 & & & 2008 & & & 2009 & \\
\hline Isolates & All & $\mathrm{U}$ & $\mathrm{NU}$ & All & $\mathrm{U}$ & NU & All & $\mathrm{U}$ & $\mathrm{NU}$ & All & $\mathrm{U}$ & $\mathrm{NU}$ & All & $\mathrm{U}$ & NU \\
\hline Number of isolates & 998 & 609 & 389 & 1072 & 711 & 361 & 1049 & 688 & 361 & 1098 & 727 & 371 & 1011 & 628 & 383 \\
\hline$\%$ of ESBL & 20.3 & 15.7 & 26.1 & 17.4 & 15.6 & 19.1 & 19.45 & 19.2 & 19.8 & 19.4 & 17.1 & 22.0 & 18.6 & 16.8 & 21.8 \\
\hline
\end{tabular}

TABle 5: Susceptibility profiles of ESBL producing E. coli collected from Lebanese patients between 2005 and 2009 at the Saint George Hospital-University Medical Center, Beirut.

\begin{tabular}{|c|c|c|c|c|c|c|c|c|}
\hline \multirow[b]{3}{*}{ Year } & \multirow[b]{3}{*}{ Isolates } & \multicolumn{7}{|c|}{ Percentages of susceptibility of ESBL producing E. coli } \\
\hline & & \multicolumn{7}{|c|}{ Antibacterial agent } \\
\hline & & Amikacin & Cefoxitin & Gentamycin & Pip + Tazo & TSM & Tigecycline & Ciprofloxacin \\
\hline \multirow{3}{*}{2005} & All & 90.5 & 85.6 & 29.8 & 97.6 & 39.4 & ND & 21.1 \\
\hline & Urinary & 92.3 & 87.6 & 35.2 & 97.5 & 41.1 & ND & 16.4 \\
\hline & Nonurinary & 88.2 & 83.5 & 25.1 & 97.8 & 37.1 & ND & 17.9 \\
\hline \multirow{3}{*}{2006} & All & 97.9 & 80.7 & 27.3 & 93.3 & 24.1 & ND & 17.6 \\
\hline & Urinary & 95.6 & 84.4 & 33.5 & 94.1 & 40.8 & $\mathrm{ND}$ & 18.8 \\
\hline & Nonurinary & 98.3 & 76.9 & 22.1 & 93.5 & 18.9 & ND & 16.5 \\
\hline \multirow{3}{*}{2007} & All & 93.6 & 81.4 & 30.1 & 92.2 & 23.5 & 89.6 & 23.1 \\
\hline & Urinary & 91.2 & 87.5 & 22.4 & 89.9 & 36.8 & 91.2 & 25.7 \\
\hline & Nonurinary & 90.2 & 75.6 & 36.8 & 95.4 & 41.3 & 93.2 & 19.1 \\
\hline \multirow{3}{*}{2008} & All & 94.2 & 80.6 & 27.8 & 94.1 & 25.6 & 87.4 & 18.8 \\
\hline & Urinary & 89.1 & 82.4 & 24.4 & 87.9 & 26.6 & 91.4 & 21.1 \\
\hline & Nonurinary & 97.3 & 77.1 & 32.2 & 98.8 & 23.9 & 82.1 & 15.5 \\
\hline \multirow{3}{*}{2009} & All & 93.6 & 83.4 & 30.8 & 94.2 & 19.5 & 91.6 & 19.6 \\
\hline & Urinary & 94.6 & 84.5 & 36.1 & 96.6 & 25.4 & 94.4 & 19.5 \\
\hline & Nonurinary & 91.8 & 82.2 & 26.3 & 92.2 & 10.6 & 89.9 & 19.2 \\
\hline
\end{tabular}

TABLE 6: Multiple resistance of urinary isolates of E. coli.

\begin{tabular}{|c|c|c|c|c|c|}
\hline \multirow[b]{2}{*}{ Resistance profile of urinary E. coli } & \multicolumn{5}{|c|}{ Percentage of resistance profiles } \\
\hline & 2005 & 2006 & 2007 & 2008 & 2009 \\
\hline Resistant to aminoglycosides and fluoroquinolones & 3.2 & 2.6 & 3.1 & 3 & 3.7 \\
\hline Resistant to 3rd-Generation cephalosporins and fluoroquinolones & 3.6 & 3 & 4.9 & 8.2 & 9.6 \\
\hline Resistant to 3rd-Generation cephalosporins and aminoglycosides & 3.9 & 3.4 & 3.5 & 2.5 & 3.1 \\
\hline $\begin{array}{l}\text { Resistant to 3rd-Generation cephalosporins, aminoglycosides, and } \\
\text { fluoroquinolones }\end{array}$ & 8.7 & 9 & 9.8 & 13.8 & 7.3 \\
\hline
\end{tabular}

Enterobacteriaceae. This was previously reported in our hospital as well as in other institutions in the country [1115]

In this study, E. coli accounted for approximately $61 \%$ of all clinically significant urinary isolates and $76.8 \%$ of all Enterobacteriaceae. This is consistent with the findings of previous studies in which E. coli was the predominant pathogen isolated from patients with UTIs $[16,17]$. The rate of isolation of Klebsiella pneumoniae might be described as high when compared to other studies $[16,17]$; however, this can be explained by the fact that our study includes both hospital and acquired UTI.

E. coli isolates from urinary infections show a similar pattern of susceptibility to those isolated from all body site 
infections although with a more enhanced susceptibility percentages. Aminopenicillins do not constitute a therapeutic option in this population; even the combination amoxicillinclavulanic acid does not seem to offer important alternative for treatment. In view of the increasing ESBL production, all cephalosporins activities are affected and challenged by these inactivating enzymes. Imipenem remains the only antibiotic with $100 \%$ of susceptibility.

It is pretty alarming to note that between 7.3 and $13.8 \%$ of the urinary isolates of $E$. coli show simultaneous resistance to third-generation cephalosporins, aminoglycosides, and fluoroquinolones. A genetic investigation on these isolates should be performed in order to identify the mechanisms of resistance and discover whether they are correlated to each other or independently occurring.

\section{Conflict of Interests}

The authors declare that they have no conflict of interests.

\section{References}

[1] A. Hoberman and E. R. Wald, "Urinary tract infections in young febrile children," Pediatric Infectious Disease Journal, vol. 16, no. 1, pp. 11-17, 1997.

[2] J. R. Delanghe, T. T. Kouri, A. R. Huber et al., "The role of automated urine particle flow cytometry in clinical practice," Clinica Chimica Acta, vol. 301, no. 1-2, pp. 1-18, 2000.

[3] K. Hryniewicz, K. Szczypa, A. Sulikowska, K. Jankowski, K. Betlejewska, and W. Hryniewicz, "Antibiotic susceptibility of bacterial strains isolated from urinary tract infections in Poland," Journal of Antimicrobial Chemotherapy, vol. 47, no. 6, pp. 773-780, 2000.

[4] M. Pezzlo, "Detection of urinary tract infections by rapid methods," Clinical Microbiology Reviews, vol. 1, no. 3, pp. 268280, 1988.

[5] M. Bonadio, M. Meini, P. Spitaleri, and C. Gigli, "Current microbiological and clinical aspects of urinary tract infections," European Urology, vol. 40, no. 4, pp. 439-445, 2001.

[6] Clinical and Laboratory Standards Institute, "Performance standards for antimicrobial susceptibility testing," CLSI document M100-S15, Clinical and Laboratory Standards Institute, Wayne, Pa, USA, 15th International supplement, 2005.

[7] N. Grude, Y. Tveten, and B. E. Kristiansen, "Urinary tract infections in Norway: bacterial etiology and susceptibility. A retrospective study of clinical isolates," Clinical Microbiology and Infection, vol. 7, no. 10, pp. 543-547, 2001.

[8] C. Kripke, "Duration of therapy for women with uncomplicated UTI," American Family Physician, vol. 72, no. 11, 2005.

[9] M. Kumar, V. Lakshmi, and R. Rajagopalan, "Occurrence of extended spectrum $\beta$-lactamases among Enterobacteriaceae spp. isolated at a tertiary care Institute," Indian Journal of Medical Microbiology, vol. 24, no. 3, pp. 208-211, 2006.

[10] N. R. Kahan, D. P. Chinitz, D. A. Waitman, D. Dushnitzky, E. Kahan, and M. Shapiro, "Empiric treatment of uncomplicated urinary tract infection with fluoroquinolones in older women in Israel: another lost treatment option?" Annals of Pharmacotherapy, vol. 40, no. 12, pp. 2223-2227, 2006.

[11] S. S. Kanj, J. E. Corkill, Z. A. Kanafani et al., "Molecular characterisation of extended-spectrum $\beta$-lactamase-producing Escherichia coli and Klebsiella spp. isolates at a tertiary-care centre in Lebanon," Clinical Microbiology and Infection, vol. 14, no. 5, pp. 501-504, 2008.
[12] Z. Daoud, C. Moubareck, N. Hakime, and F. DoucetPopulaire, "Extended spectrum $\beta$-lactamase producing enterobacteriaceae in lebanese ICU patients: epidemiology and patterns of resistance," Journal of General and Applied Microbiology, vol. 52, no. 3, pp. 169-178, 2006.

[13] C. Moubareck, Z. Daoud, N. I. Hakimé et al., "Countrywide spread of community- and hospital-acquired extendedspectrum $\beta$-lactamase (CTX-M-15)-producing Enterobacteriaceae in Lebanon," Journal of Clinical Microbiology, vol. 43, no. 7, pp. 3309-3313, 2005.

[14] G. M. Matar, S. Al Khodor, M. El-Zaatari, and M. Uwaydah, "Prevalence of the genes encoding extended-spectrum $\beta$ lactamases, in Escherichia coli resistant to $\beta$-lactam and non- $\beta$-lactam antibiotics," Annals of Tropical Medicine and Parasitology, vol. 99, no. 4, pp. 413-417, 2005.

[15] Z. Daoud and N. Hakime, "Prevalence and susceptibility patterns of extended-spectrum betalactamase-producing Escherichia coli and Klebsiella pneumoniae in a general university hospital in Beirut, Lebanon," Revista Espanola de Quimioterapia, vol. 16, no. 2, pp. 233-238, 2003.

[16] C. A. McNulty, J. Bowen, G. Clark, A. Charlett, and K. Cartwright, "How should general practitioners investigate suspected urinary tract infection? Variations in laboratoryconfirmed bacteriuria in South West England," Communicable Disease and Public Health, vol. 7, no. 3, pp. 220-226, 2004.

[17] G. Kahlmeter, "Prevalence and antimicrobial susceptibility of pathogens in uncomplicated cystitis in Europe. The ECO. SENS study," International Journal of Antimicrobial Agents, vol. 22, no. 2, pp. S49-S52, 2003. 


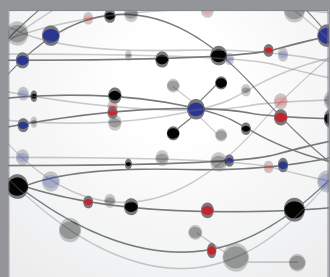

The Scientific World Journal
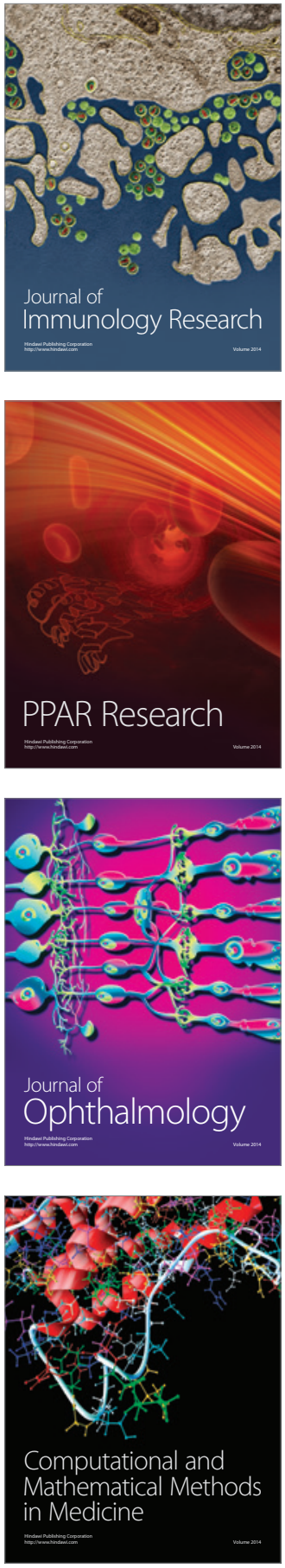

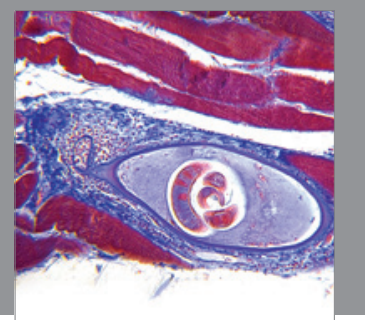

Gastroenterology

Research and Practice
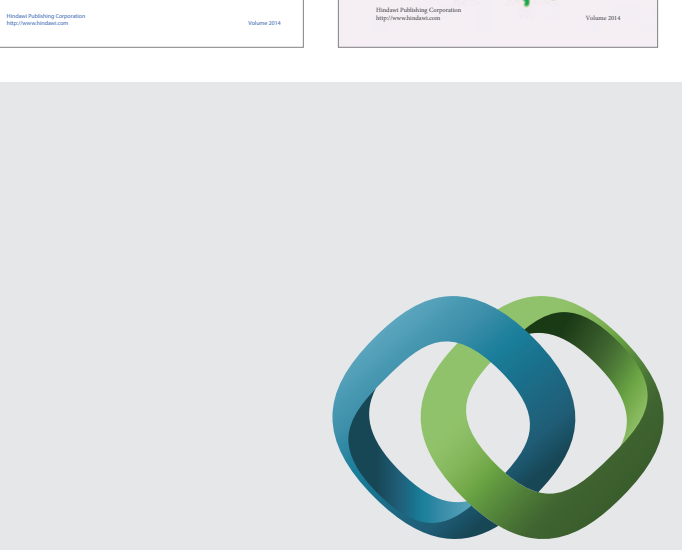

\section{Hindawi}

Submit your manuscripts at

http://www.hindawi.com
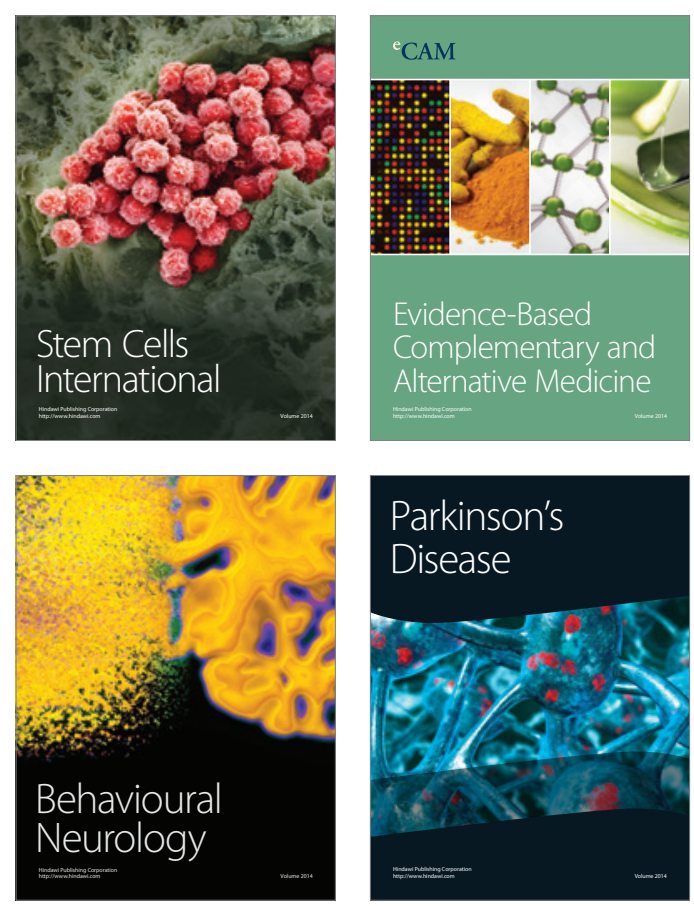

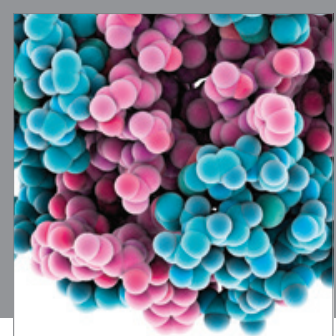

Journal of
Diabetes Research

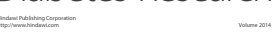

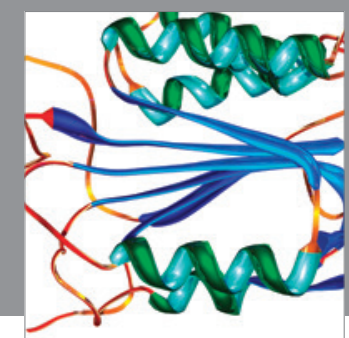

Disease Markers
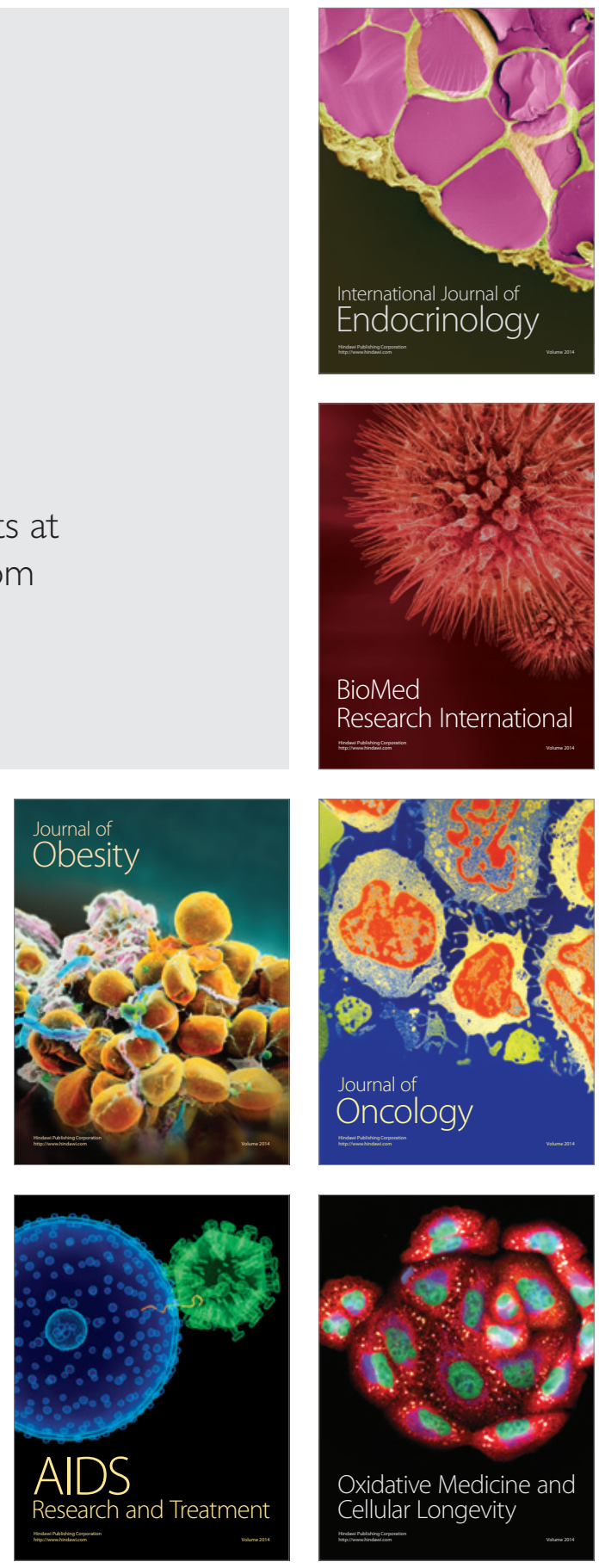